\title{
Shadow Detection and Removal from Remote Sensing Images Using NDI and Morphological Operators
}

\author{
Krishna Kant Singh \\ Earthquake Engineering \\ IIT Roorkee, Roorkee, India
}

\author{
Kirat Pal \\ Earthquake Engineering \\ IIT Roorkee, Roorkee, India
}

\author{
M.J.Nigam \\ E\&C Department \\ IIT Roorkee, Roorkee, India
}

\begin{abstract}
Shadows appear in remote sensing images due to elevated objects. Shadows cause hindrance to correct feature extraction of image features like buildings ,towers etc. in urban areas it may also cause false color tone and shape distortion of objects, which degrades the quality of images. Hence, it is important to segment shadow regions and restore their information for image interpretation. This paper presents an efficient and simple approach for shadow detection and removal based on HSV color model in complex urban color remote sensing images for solving problems caused by shadows. In the proposed method shadows are detected using normalized difference index and subsequent thresholding based on Otsu's method. Once the shadows are detected they are classified and a non shadow area around each shadow termed as buffer area is estimated using morphological operators. The mean and variance of these buffer areas are used to compensate the shadow regions.
\end{abstract}

\section{KEYWORDS}

shadow compensation, shadow detection, thresholding.

\section{INTRODUCTION}

In urban remote sensing images, shadows are usually cast by elevated objects such as various cultural features (buildings, bridges, towers, etc.) when they are illuminated by the Sun at the time of exposures. Shadow in remote sensing image is conflict information in computer image processing. On the one hand, it can reduce the successful rate of edge extraction, object recognition, image matching, change detection and other processing for the corresponding ground objects in the shadow $[1,2]$. On the other hand, it can produce a great deal of useful information about shape, relative position, surface character and other characters of the object generating shadow $[3,4,5]$. It is a necessary step to eliminate shadow and restore the scenes in the shadow area before performing object recognition and image matching tasks for the shadow area.Thus, shadow detection and elimination has become very important in image processing. Shadow detection and removal has wide application in change detection from remote sensing images done to assess damage due to natural disasters like earthquakes, tsunamis, landslides etc. since shadows obstruct the correct extraction of buildings and shadows lead to false detections. VHR remote sensed images show very fine details of features such as building, roads, vehicles, and trees.
However, the amount of shadow increases with the spatial resolution. Shadow detection and removal methods work together to remove shadows. Shadow detection methods can be categorized into method based on model and method based on shadow property [7][8][9][10][11].Shadow spectra and shadow geometrical features are used in shadow property based methods [9] [12]. Tsai presented a method which uses the spectral ratio image in HSI space to segment shadow[10]; Polodorio proposed a method by thresholding saturation intensity difference image in HSI color space[13].

Shadow compensation is to restore the surface under shadows. Considering that a surface texture does not significantly change when shadowed, neighboring non shadowed segments are usually used to compensate shadowed ones. In the literatures, algorithms include gamma correction method [14], linear correlation method [15], posteriori probabilities method [16].Almost all these approach are operated band by band in RGB color space, which makes the composite color unnatural [17].Another method is proposed in [6].To overcome the above mentioned problems, a simple and efficient shadow detection and removal algorithm for remote sensing images is proposed in this paper. The proposed method first detects shadow by considering the HSV color space using Otsu's method of thresholding. Once shadows are detected they are removed using the mean and variance values of the buffer area which is the non shadow area around each shadow.

\section{HSV MODEL}

HSV model has the following relations with RGB model:

$V=\frac{1}{3}(R+G+B)$

$S=1-\frac{3}{R+G+B} \min (R, G, B)$

$H=\left\{\begin{array}{lll}\theta & \text { if } & B \leq G \\ 360^{0}-\theta & \text { if } & B>G\end{array}\right.$

in which

$\theta=\cos ^{-1}\left\{\frac{\frac{1}{2}[(R-G)+(R-B)]}{\sqrt{(R-G)^{2}+(R-B)(G-B)}}\right\}$ 


\section{PROPOSED WORK}

In the proposed method shadow is first detected using HSV color model and then it is removed using a buffer area around the shadow region. The approach used in this paper is shown in fig. 1 An RGB image with shadows is given as input. This input image is converted from RGB to HSV space as HueSaturation-Value (HSV) color space is invariant to shadow i.e., it conveys the spectral or color characteristics of image features, regardless of variations in scene illumination condition [18]. Shadow segmentation is done in HSV color space using Otsu's method of thresholding this separates shadows and non shadows. For compensation of shadowed region the HSV color space is used, a buffer area around each shadow is computed and using this buffer area the new values for shadowed regions are computed. Each of these steps are discussed in the next sub sections

\section{A. Shadow Segmentation}

The input image is first converted to HSV color space using equations (1),(2),(3) and (4) and is denoted by $I$. It has been observed that shadow areas are dark and saturated strongly with the blue and violet wavelength. In HSV color space, shadows hold some different spectral properties as follows $[10,13,19]$ :

1) low value because the direct light from the Sun is occluded by elevated objects;

2) high saturation with short blue-violet wavelength due to atmospheric Rayleigh scattering effect;

3) high hue values because shadow areas are dark.

Hue is an attribute associated with the dominant wavelength in a mixture of light waves. Hue represents dominant color as perceived by an observer. Saturation refers to the relative purity or the amount of white light mixed with hue. Thus, shadow areas have maximum value of saturation and the minimum value component in HSV color space. Some other features like vegetation, roads etc. have similar characteristics. The $\mathrm{S}$ and $\mathrm{V}$ components of $I$ are used to extract shadows using NDI as,

$N D I=\frac{s-V}{s+V}$

In order to detect shadow areas, a positive threshold $T$ can be found to segment the NDI images. For automatic computation of threshold OTSU thresholding algorithm is used. The Otsu's method finds an optimal threshold T, which maximizes [8]

$\operatorname{NDI}(T)=\frac{(\bar{\mu} \cdot w(T)-\mu(T))^{2}}{w(T) \cdot \mu(T)}$

where $w(T)=\sum_{i=0}^{T} p_{i}, \mu(T)=\sum_{i=T+1}^{255} p_{i}, \bar{\mu}=\sum_{i=0}^{255} i \cdot p_{i}$ and $p_{i}$ is the probability of pixels with grey level $i$ in the image. The Image pixels which have a higher NDI than the threshold (T) are accepted as shadow pixel; otherwise not.

$$
I_{\text {shadow }}(i, j)= \begin{cases}1 & N D I(i, j) \geq T \\ 0 & N D I(i, j)<T\end{cases}
$$

The image obtained after this thresholding will be a binary image with all shadow pixels set to 1 and all non shadow pixels are set to 0 .This binary image is denoted by $\mathrm{I}_{\text {shadow }}$.

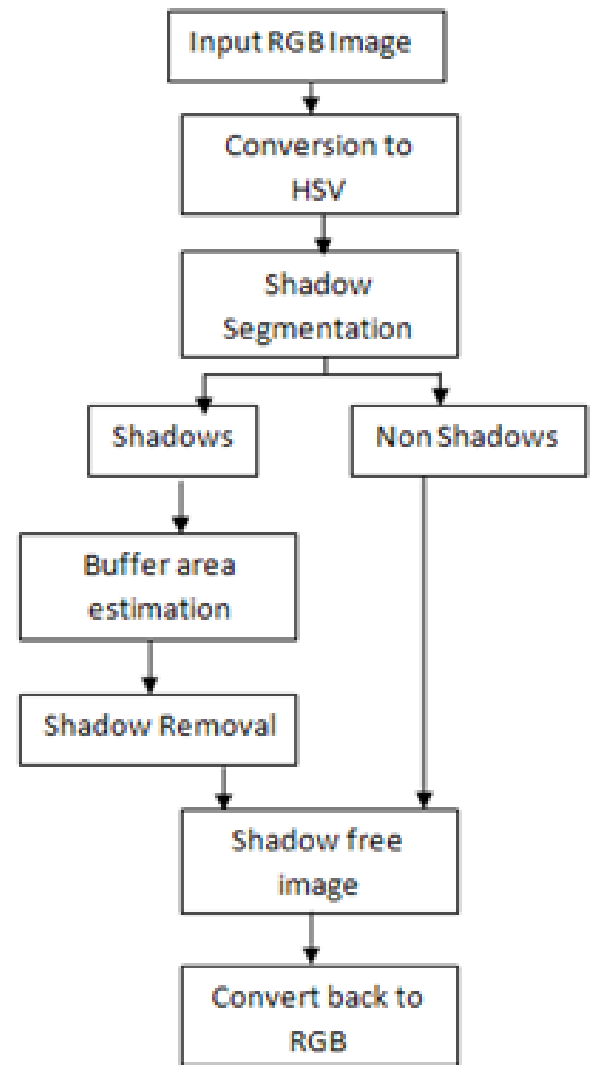

Fig.1Approach used in the proposed method.

\section{B. Shadow Removal}

In the proposed method shadow is removed, using a buffer area around the shadow. The buffer area is used to compensate the shadow using the mean and variances of the buffer area and the shadow region. The buffer area is estimated using morphological operations on $\mathrm{I}_{\text {shadow }}$. The steps involved are as follows:

Step1: Shadow Classification

First, shadows are classified based on the concept of connected components. The binary image $\mathrm{I}_{\text {shadow }}$ has one or more connected components(each connected region corresponds to a shadow), an array $\mathrm{I}_{0}$ (of the same size as $\left.\mathrm{I}_{\text {shadow }}\right)$ whose elements are $0 \mathrm{~s}$, except at each location known to correspond to a point in each connected component in $\mathrm{I}_{\text {shadow }}$ is set to 1 .The following iterative procedure finds all connected components:

$I_{n}=\left(I_{n-1} \oplus B\right) \cap I_{\text {shadow }} \quad n=1,2,3 \ldots$

where $\mathrm{B}$ is a suitable structuring element. The procedure terminates when $I_{n}=I_{n-1}$, with $I_{n}$ containing all connected components of $\mathrm{I}_{\text {shadow. }}$. This process yields $m$ sets of connected components or $m$ different shadows in the image. $m$ can have the value one or greater than one, depending on the number of shadows.

Step 2: Buffer Area Estimation

Buffer area of each shadow is the non shadow area around that shadow. Thus, after classifying the shadows using eq (8), the buffer area of each shadow is computed using morphological dilation operation and image subtraction operation as follows:

$$
I_{\text {dilated }, k}=\left(I_{k} \oplus B_{\text {square }}\right)
$$


The dilation operation will expand the shadow boundaries. $\mathrm{B}_{\text {square }}$ is a square structuring element. The size of the structuring element will decide the size of buffer area. In this paper a $3 \times 3$ square structuring element is used.

$I_{\text {buff }, k}=\left(I_{\text {dilated }, k}-I_{k}\right)$

where $\mathrm{k}=1,2, \ldots . \mathrm{m}$ and $I_{b u f f, k}$ gives the location of the non shadow points, i.e, the buffer area around each shadow $k$.

\section{Step 3 Shadow removal}

The shadow is removed using the following transformation function.

$$
I_{k}^{\prime}(i, j)=\mu_{b u f f, k}+\frac{I_{k}(i, j)-\mu_{k}}{\sigma_{b u f f, k}} . \sigma_{k}
$$

where $\mathrm{k}=1,2, \ldots . \mathrm{m}$.

$I_{k}^{\prime}(i, j)$ is the compensated value of the shadow pixel.

$\mu_{b u f f, k}$ and $\sigma_{b u f f, k}$ are the mean and variance of the pixels of image $I$ at locations $I_{b u f f, k}$.

$\mu_{k}$ and $\sigma_{k}$ are the mean and variance of the shadow pixels of image $I$ at locations $I_{k}$. The resulting image is shadow free image.

\section{EXPERIMENTAL RESULTS}

The above method was tested in Matlab R2009a using three different RGB images. Fig. 2(a) shows a remote sensing RGB image of an urban area with buildings it contains shadows cast by the elevated objects, i.e., buildings. Fig.2(b) shows the shadows detected, the shadows are shown in white colour. The buffer area around each shadow is shown in fig.2(c).It can be seen that the shadows are removed quite efficiently as shown in fig.2(d).

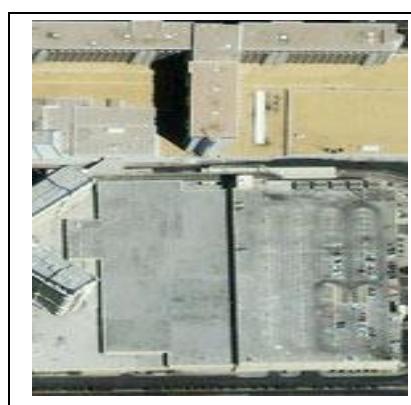

(a)

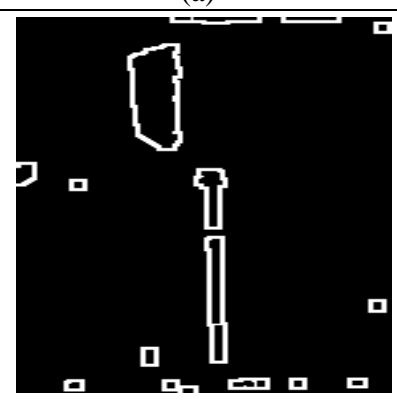

(c)

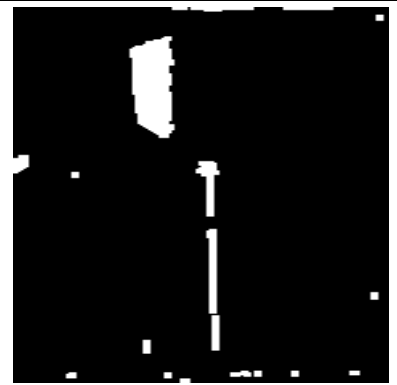

(b)

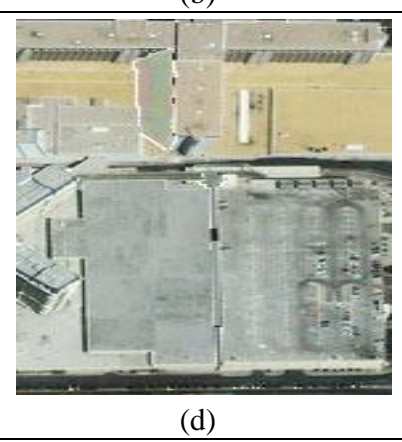

(d)
Fig. 2(a) RGB image (b) Binary image showing shadows (c) Buffer area of shadows (d) Shadow free image.

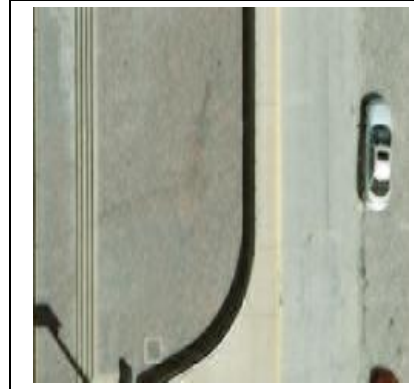

(a)

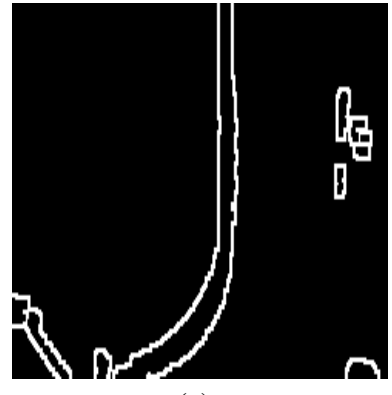

(c)

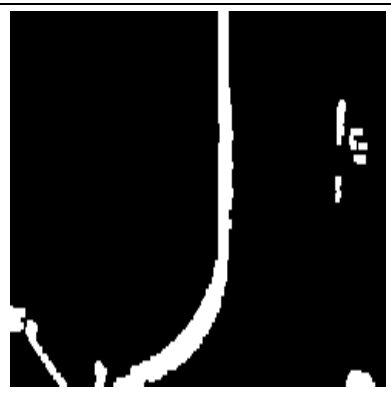

(b)

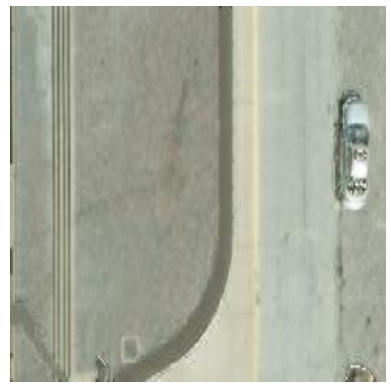

(d)
Fig. 3(a) RGB image (b) Binary image showing shadows (c) Buffer area of shadows (d) Shadow free image.

Fig. 3(a) shows a remote sensing RGB image of a highway with vehicles it contains some shadows. Fig.3(b) shows the shadows detected, the shadows are shown in white color. The accuracy of shadow detection can be seen from the fact that roads are not detected as shadows, though they have similar characteristics as shadows. The buffer area around each shadow is shown in fig.3(c).The shadow free image is shown in fig.3(d) where only the shadows are removed and the road remains unaffected. Fig. 4(a) shows a remote sensing RGB image of a vehicle covered by a shadow and thus, the vehicle is not properly visible. Fig. 4(b) shows the shadows detected, the shadows are shown in white color. Fig.4(c) shows the buffer area. The area under the shadow is illuminated in fig.4(d) and the vehicle is now clearly visible. The results show the effectiveness of the proposed method in deshadowing as it can be seen in the results that the area under the shadows is revealed in the shadow free image.

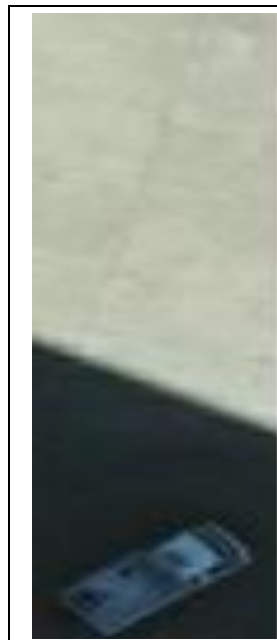

(a)

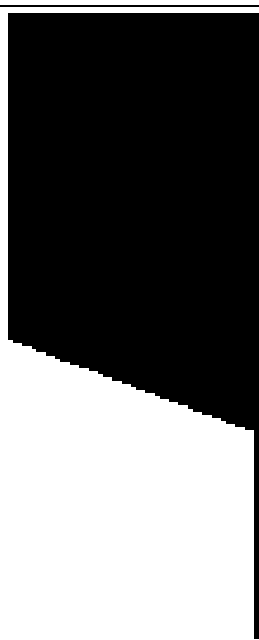

(b) 


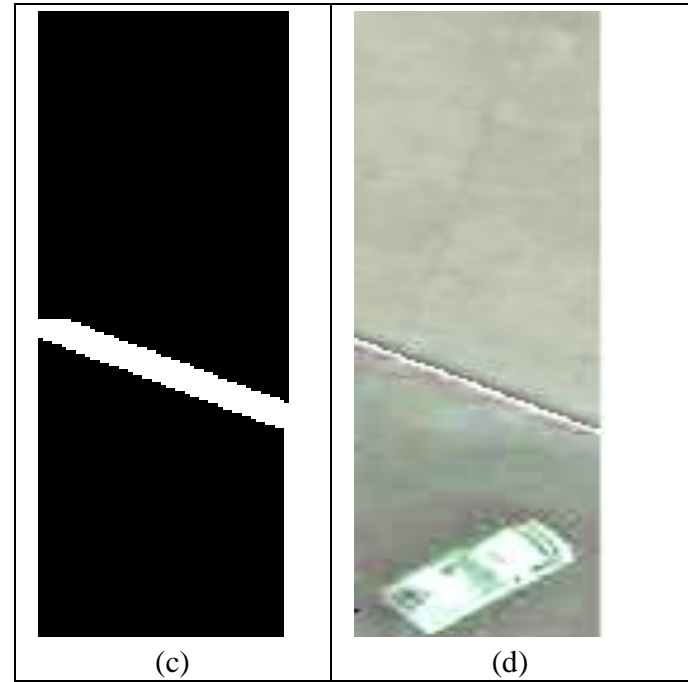

Fig. 4(a) RGB image (b) Binary image showing shadows (c) Buffer area of shadows (d) Shadow free image

\section{CONCLUSION}

In this paper an efficient method for shadow detection and removal from remote sensing images is proposed. The method works in HSV space to detect shadows and then uses Otsu's thresholding method to detect shadows. After the shadows are detected they are removed by using the mean and variance value of the buffer area around each shadow. The buffer area is estimated with the morphological operators. The results are quite effective and it is seen that shadows are detected correctly. The shadow free image is also quite effective and the areas under the shadow are illuminated.

\section{REFERENCES}

[1] Wang Junli, Wang Shugen, 2002. A method of image shadow detection based RGB colour space. Information Technology, 26(12), pp.7-9.

[2] Yan Li,Tadashi Sasagawa and Peng Gong,2004. A System of the Shadow Detection and Shadow Removal for High Resolution City Aerial Photo. ISPRS, COMMISSION III, ISTANBUL.

[3] Barnes N.and Liu Z.Q,1999. Knowledge-based shape from shading. Int. J. of Pattern Recognition and Artificial Intelligence, 13(1), pp.1-23.

[4] Ortega A.and Shah M, 1998. From shape from shading to object recognition. Int. J. of Pattern Recognition and Artificial Intelligence, 12(2), pp.191-208.

[5] Yang Yijun, Zhao Rongchun and Jiang Wenbing, 2002. Detection of shadow areas from aerial imagery. Signal Processing,18(3),pp.228-232.

[6] Wang, Ning ; Lang, Congyan ; Xu, De ,2011, ImageBased Shadow Removal via Illumination Chromaticity Estimation in Multimedia Information Networking and Security (MINES),pp. 33-36
[7] Y. Li, P. Gong, T. Sasagawa, Integrated shadow removal based on photogrammetry and image analysis. International Journal of Remote Sensing, vol. 26(18):pp. 3911-3929, 2005.

[8] N. Otsu, A threshold selection method from gray level histograms. IEEE Trans. Syst., Man, Cybern, vol,9 (1) : pp. 62-69,1979

[9] J. Huang, W. Xie and L. Tang, Detection of and compensation for shadows in colored urban aerial images. Hangzhou, China: in Proc. $5^{\text {th }}$ World Congr. Intelligent Control and Automation, pp. 3098-3100. 2004.

[10] V.J.D. Tsai, A comparative study on shadow compensation of color aerial images in invariant color models. IEEE Trans. On Geoscience and Remote Sensing, vol. 44(6): pp.1661-1671, 2006.

[11] Y. Li, T. Sasagawa and O. Gong, A system of the shadow detection and shadow removal for high resolution city aerial photo. ISPRS,Commision III, Istanbul, 2004.

[12] M. Nagao, T. Matsutyama and Y. Ikeda. Region extraction and shape analysis in aerial photographs, Computer Vision, Graphics and Image Process, vol. 10(3): pp. 195-223, 1979.

[13] Polodorio, A.M., Flores, F.C., Imai, N.N., Tommaselli, A.M.G., and Franco, C. "Automatic shadow segmentation in aerial color images", Proceedings of XVI Brazilian symposium on computer graphics and image processing, 270-277, 2003.

[14] Massalabi, A., He, D.C., Bénié, G.B., and Beaudry, E."Detecting information under and from shadow in panchromatic Ikonos images of the city of sherbrooke", IGARSS 2004, vol.3, 2000-2003, 2004.

[15] Sarabandi, P., Yamazaki, F., Matsuoka, M., and Kiremidjian, A. "Shadow detection and radiometric restoration in satellite high resolution images", IGARSS 2004, vol.6, 3744- 3747, 2004.

[16] Suzuki, A., Shio, A., Arai, H., and Ohtsuka, S. "Dynamic shadow compensation of aerial images based on color and spatial analysis", Proc.15th International Conference on Pattern Recognition, 317-320, 2000.

[17] Su, J., Lin, X.G., Liu, D.Z. "An automatic shadow detection and compensation method for remote sensed color images", The 8th International Conference on Signal Processing, 2006.

[18] Thomas M. Lillesand and Ralph W. Kiefer, "Remote Sensing and Image Interpretation", Fourth Ed., John Wiley \& Sons, 2000.

[19] Su, J., Lin, X.G., Liu, D.Z. "An automatic shadow detection and compensation method for remote sensed color images", The 8th International Conference on Signal Processing, 2006. 